\title{
Produto Mínimo Viável para Monitoramento Elétrico em Smart Homes
}

\author{
Valdenir S. Severino ${ }^{1}$, Danilo R. Vasconcelos ${ }^{1}$, Ronaldo T. P. Milfont ${ }^{1}$, \\ Rossana M. C. Andrade ${ }^{1}$, Ismayle S. Santos ${ }^{1}$, Cassio T. C. Andrade ${ }^{2}$, Jose E. V. Filho ${ }^{2}$ \\ ${ }^{1}$ Mestrado e Doutorado em Ciência da Computação \\ Universidade Federal do Ceará (UFC) - Fortaleza - CE - Brasil \\ ${ }^{2}$ Centro de Ciências e Tecnologias \\ Universidade de Fortaleza (UNIFOR) - Fortaleza - CE - Brasil \\ \{rossana, valdenirseverino, ismaylesantos\}@great.ufc.br \\ \{ronaldo.milfont, danilo.vasconcelos\}egreat.ufc.br \\ \{cassiotca, euricovasconcelos\}@unifor.br
}

\begin{abstract}
Smart Home Energy Management Systems are powerful tools for efficient energy management. In this scenario, monitoring is done by reading information generated by intelligent home appliances. However, it is not every home that owns such equipment, which makes management difficult. In this work, we present a minimum viable product (MVP) of a residential electrical monitoring system capable of extracting information about the energy consumption of common household appliances, through a single point of collection and through consumer signatures to identify these appliances when they are connected to the electrical network.
\end{abstract}

Resumo. Sistemas de monitoramento de energia elétrica em Smart Homes são poderosas ferramentas para uma gestão energética eficiente. Nesse cenário, o monitoramento é feito a partir da leitura de informações geradas por eletrodomésticos inteligentes. No entanto, não são todas as casas que possuem esses equipamentos, o que dificulta o gerenciamento. Neste trabalho é apresentado um produto mínimo viável de um sistema de monitoramento elétrico para uso residencial, em que é possível extrair informações sobre o consumo energético de eletrodomésticos comuns, utilizando apenas um único ponto de coleta. Além disso, as assinaturas de consumo permitem fazer a identificação desses eletrodomésticos quando estes estão conectados à rede elétrica.

\section{Introdução}

O desperdício de energia é um dos maiores problemas enérgicos enfrentados no país. Dessa forma se torna cada vez mais necessário a utilização e desenvolvimento de estratégias voltadas para um melhor controle, economia e monitoramento do uso de energia. Do ponto de vista da demanda energética, o gerenciamento de energia consiste no método que requer menor investimento ao sistema elétrico, dado que se aplica com a racionalização do uso de energia elétrica. Ademais, apresenta rápidos resultados em curto prazo [Andreoli 2005]. Portanto, é possível inferir que essa técnica é adequada quando se 
deseja resolução rápida e com baixo investimento, especialmente quando se considera o nível de complexidade atual dos sistemas de distribuição de energia.

Neste contexto, as redes elétricas do tipo Smart Grid se apresentam como a integração de tecnologias de informação e comunicação na rede elétrica tradicional para torná-la mais inteligente e rápida na tomada de decisões [Khan et al. 2013]. Este tipo de rede proporcionou o surgimento das Smart Homes, em que residências comuns são dotadas de dispositivos inteligentes, que permitem o monitoramento e controle, de forma remota, dos principais equipamentos dentro da residência [Pilloni et al. 2018]. Além disso, existem sistemas de gerenciamento específico para esta tecnologia. Os SHEM, do inglês Smart Home Energy Management, consistem de aplicações específicas para o controle, supervisionamento e aquisição de dados referentes à gestão de energia residencial. $\mathrm{O}$ objetivo maior desse tipo de sistema é de reduzir o consumo de eletricidade e garantir um menor desperdício de energia. A partir da utilização de sistemas inteligentes, controlados por uma interface homem-máquina e das preferências do usuário, é possível monitorar, em tempo real, o consumo de vários eletrodomésticos [Pau et al. 2017].

A utilização de sistemas para gerenciamento energético nas residências possibilita benefícios para todos os atores envolvidos. Para o consumidor final temos a redução do valor da conta de energia, já para o fornecedor ocorre uma diminuição na quantidade de picos na demanda energética. Por fim, para o meio ambiente o uso deste tipo de sistema resulta em menor poluição ambiental [Nilsson et al. 2018]. No entanto, é possível perceber uma grande premissa desse tipo de sistema: a necessidade dos eletrodomésticos residenciais comuns possuírem sensores ou qualquer outra fonte de obtenção de informação o que nem sempre é viável dado o custo de tais equipamentos.

Dessa forma, este trabalho apresenta um produto mínimo viável de um sistema de monitoramento inteligente de consumo energia de forma não invasiva. A estratégia sugerida consiste na análise das componentes harmônicas obtidas na rede elétrica residêncial, permitindo assim a identificação de quais aparelhos estão ligados, além de extrair informações referentes ao consumo energético de uma residência, tudo isso com apenas um único ponto de coleta. Esse sistema tem como objetivo principal viabilizar a incorporação de aparelhos comuns em um sistema de gerenciamento de energia inteligente. Além disso, todas as informações coletadas serão disponibilizadas através de uma aplicação móvel, permitindo assim um maior controle por parte do usuário.

O restante do artigo é dividido como segue. Na Seção 2 são apresentados os trabalhos relacionados. O produto viável mínimo e a metodologia utilizada na proposta são descritos na Seção 3. A Seção 4 explica os experimentos realizados e os resultados obtidos. Por fim, na Seção 5 são apontadas as conclusões e trabalhos futuros.

\section{Trabalhos Relacionados}

Diversos trabalhos científicos têm sido desenvolvidos e publicados na área de monitoramento energético. Esta seção apresenta os trabalhos de [Siqueira 2014], [Santos et al. 2016], [Ramos and Andrade 2015] e [Lin and Chen 2017].

[Siqueira 2014] propõe o uso de uma plataforma Arduíno como central de monitoramento de consumo de energia elétrica. A proposta desse autor consiste em um sensor de corrente não-invasivo ligado a uma placa Arduíno. Nesta placa são realizados os cálculos 
dos valores de corrente, tensão, potência, consumo em KWh, tempo de medição e consumo acumulado. E a partir dos dados gerados, é criado um arquivo de relatório utilizando o Excel. Neste trabalho não é feito monitoramento do consumo elétrico em tempo real.

A solução de [Santos et al. 2016] baseia-se no uso de uma plataforma Arduíno para a aquisição das informações recebidas pelo sensor de corrente, realiza os cálculos matemáticos para determinar o consumo de energia e envia os dados por meio do módulo Wi-Fi ESP8266 a um servidor web que por meio de uma aplicação JAVA disponibiliza ao proprietário da residência os dados de consumo em forma de gráficos. Esta solução, assim como a anterior, não contempla nenhuma forma de informar qual equipamento está ligado na rede elétrica.

O trabalho de [Ramos and Andrade 2015] é bem similar ao projeto de [Siqueira 2014] quanto às especificações técnicas em relação a proposta tema deste trabalho. A proposta de [Ramos and Andrade 2015] diferencia-se pela implementação extra de um monitoramento de vazão de água. A principal desvantagem destas propostas apresentadas consiste em não disponibilizar as informações de forma detalhada e em tempo real.

A proposta de [Lin and Chen 2017] combina o uso de um medidor e tomadas inteligentes, smartphones e um servidor de banco de dados para criar um sistema de monitoramento de energia elétrica para Smart Homes. O medidor inteligente consiste de uma unidade de armazenamento e de medição de energia e um módulo ZigBee. A tomada inteligente é composta por uma unidade central de controle e um módulo de monitoramento remoto. O usuário pode usar o smartphone para verificar e controlar o funcionamento do dispositivo, e as informações que consomem energia podem ser monitoradas remotamente via Wi-Fi. É utilizado um algoritmo baseado em Support Vector Machines (SVM) para identificar o modo de operação dos eletrodoméstico monitorados. A desvantagem da abordagem utilizada nessa solução é que para cada ponto monitorado exige um hardware para tal, tornando-a cara se comparada a proposta deste trabalho.

\section{Produto Mínimo Viável}

Fundamentados nos desafios expostos na seção anterior, neste trabalho é realizado um estudo de viabilidade de um sistema de monitoramento (i.e., um MVP - Minimum Viable Product) que possa realizar, de forma não invasiva, a leitura das variáveis elétricas relativas ao consumo energético de uma residência e identificar quais os equipamentos elétricos estão em funcionamento. Dessa forma, o proprietário ou outro morador da casa poderá gerenciar de forma eficiente seus equipamentos elétricos, possibilitando a prevenção de eventuais desperdícios de energia.

O sistema de monitoramento proposto consiste em três módulos: (i) módulo de hardware, responsável pela aquisição de dados, pré-processamento e envio das informações para a Web; (ii) serviço Web em nuvem, responsável pela recepção das informações enviadas pelo módulo de hardware e pelo processo de identificação de eletrodomésticos; e (iii) aplicação móvel Android, na qual o proprietário ou outro morador da residência poderá fazer o monitoramento de seu consumo energético. 


\subsection{Módulo de Hardware para aquisição de dados}

De forma a minimizar tamanho e custo, foi utilizado o Mini Breakout Kit Intel Edison para executar a aquisição de dados, juntamente de dois sensores, um para leitura da tensão da rede e outro para obtenção da corrente. A escolha dessa ferramenta se baseou no fato de ser um dispositivo inteligente e robusto, contendo módulos de comunicação já presentes no próprio chip e um sistema operacional Linux embarcado, facilitando assim o desenvolvimento das aplicações responsáveis pelo envio e processamento das informações coletadas. Para reduzir a complexidade envolvida em manipular os sinais de tensão e corrente diretamente, a comunicação entre o Edison e o sensores acontece através de uma placa de áudio conectada via USB.

Segundo a [ABNT 2004], o padrão de frequência da rede elétrica de baixa tensão, o mais comum em residências, é de 60 hertz. Dado que este sinal é contínuo no tempo e em nível, é necessário transmitir apenas uma certa quantidade de amostras deste sinal. Neste trabalho utiliza-se como base o Teorema de Nyquist, no qual a quantidade mínima de amostras a serem obtidas de um sinal amostrado deve ser duas vezes maior que a frequência deste sinal, permitindo sua recuperação [Nyquist 1928]. Logo, para a medição de um sinal de 60 hertz é necessário analisar, no mínimo, 120 amostras por segundo. O número de bits de cada amostra depende da qualidade do conversor A/D usado, considerando um conversor de 8 bits, por exemplo, a taxa mínima de bits necessária para reconstruir o sinal original é de 960 bits por segundo. A placa de áudio USB utilizada possibilita uma aquisição de dados com uma taxa de 48000 bits por segundo por canal. Essa taxa de aquisição possibilita a reconstrução mais precisa do sinal medido pelo canal. Uma vez o hardware de aquisição de dados é capaz de analisar cerca de 800 amostras por segundos.

O sistema operacional Linux possui uma biblioteca nativa para captura de áudio nativa chamada ALSA. A partir do uso da API disponibilizada pela biblioteca, o firmware de desenvolvimento faz a aquisição de dados em lotes de 800 amostras por vez. Cada amostra possui dois valores, um referente ao canal direito (sensor de tensão) e o outro ao canal esquerdo (sensor de corrente). Esses valores correspondem a números decimais originados da digitalização do sinal analógico na entrada do conversor analógico-digital (A/D) interno da placa de áudio e são utilizados para calcular os valores das principais medidas relacionadas ao consumo energético: tensão eficaz, corrente eficaz, potência real, potência instantânea, potência aparente e o fator de potência.

A potência instantânea representa a velocidade com que se consome ou se absorve energia em um instante de tempo [Alexander and Sadiku 2013]. A partir dessa medida elétrica são calculadas as demais grandezas relacionadas ao consumo energético. A potência real representa a quantidade média de energia consumida por uma carga ao longo de um período de tempo. A tensão eficaz pode ser entendida como a tensão alternada equivalente a uma tensão contínua para um circuito cuja potência gasta é a mesma nos dois casos (alternada e contínua). Sua obtenção é feita a partir do cálculo da raiz quadrada da média dos quadrados de cada elemento de tensão instantânea durante um período. De forma semelhante acontece o cálculo da corrente eficaz. A potência aparente representa a potência total fornecida pela fonte e é obtida através da multiplicação da tensão eficaz pela corrente eficaz. Por fim, o fator de potência é a razão entre potência real e potência aparente. Trata-se de uma medida que indica a eficiência com a qual a energia está sendo 
usada. Um alto fator de potência indica uma eficiência alta e inversamente um fator de potência baixo indica baixa eficiência. $\mathrm{O}$ fator de potência é determinado pelo tipo de carga ligada ao sistema elétrico, que pode ser: resistiva, indutiva ou capacitiva.

Na Figura 1 é ilustrado como o equipamento é instalado na residência. Todos os componentes do módulo de hardware são encapsulados em um invólucro de proteção com um LED RGB e instalado próximo ao quadro de luz da residência.

Figura 1. Representação de aquisição de dados.

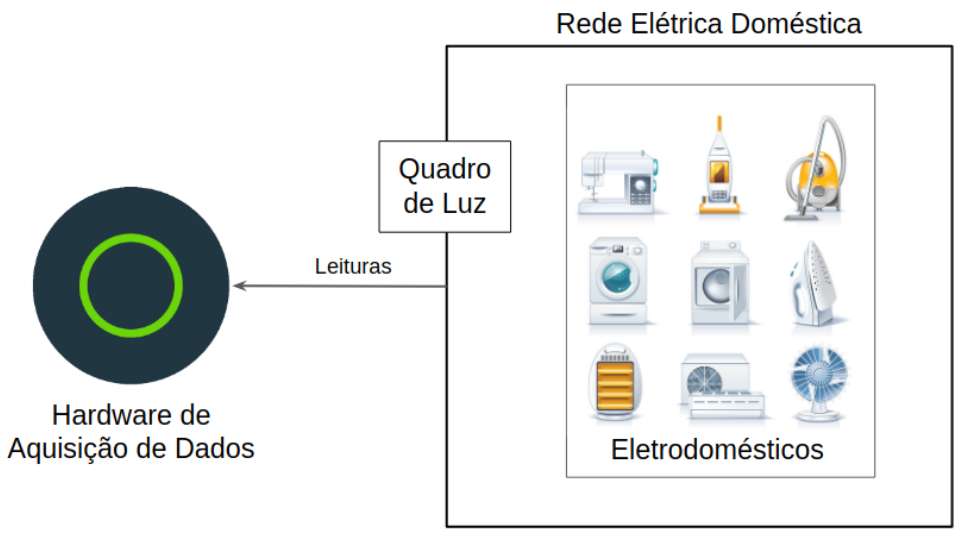

A utilização do LED RGB permite que sejam gerados feedbacks visuais indicando o nível de consumo energético instantâneo a partir de cores específicas: verde para ausência de consumo, amarelo indicando consumo presente e vermelho para consumos acima de $1000 \mathrm{~W}$. O valor para a faixa de alto consumo pode ser alterado pelo usuário através do aplicativo diretamente pelo smartphone.

\subsection{Protocolo de comunicação}

Para realizar a comunicação com o serviço Web na nuvem foi escolhido o protocolo MQTT, este protocolo é bastante utilizado no contexto de Internet das Coisas (IoT) devido à sua facilidade de implementação em diversas linguagens e bem aceito pelo mercado [Jaffey 2014].

O protocolo MQTT segue o modelo cliente/servidor. Para a troca de mensagens é utilizado o modelo publish/subscriber (publicador/subscritor). As mensagens a serem transmitidas são publicadas para um endereço, de forma semelhante a uma estrutura de diretórios em um sistema de arquivos, por exemplo: casa/sensor/temperatura [Jaffey 2014].

Na Figura 2 é apresentado o padrão de comunicação MQTT utilizado, na qual o módulo de hardware descrito anteriormente envia as publicações contendo as informações de consumo energético. Estas publicações são tratadas pelo serviço Web em nuvem, este atua como um Broker, fornecendo acesso às informações coletadas para os clientes MQTT.

\subsection{Serviço de Nuvem e Classificação dos Equipamentos Elétricos}

Para a implementação do servidor ou Broker, seguindo a filosofia do protocolo MQTT, utilizamos a ferramenta Node-RED executada em uma máquina virtual Ubuntu e instalada 
Figura 2. Sistema de troca de mensagens seguindo o protocolo MQTT

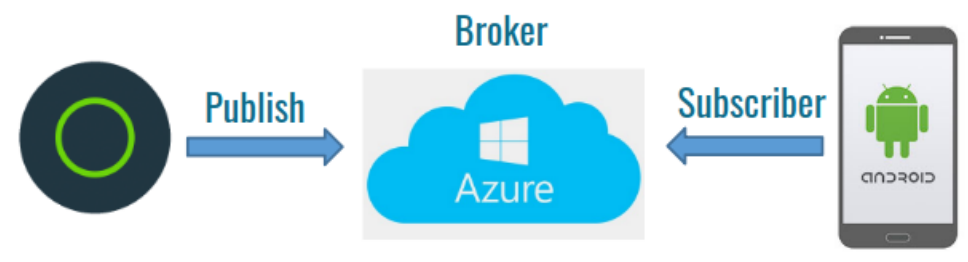

na plataforma de nuvem Microsoft Azure. O Node-RED foi escolhido por oferecer diversas ferramentas para criação de dashboards, manipuladores de banco de dados, interfaces de $e$-mails, suporte a diversos protocolos de comunicação, além de funções personalizadas.

Na Figura 3 são ilustrados dois fluxos criados a partir do Node-RED. O primeiro fluxo é responsável por receber as informações geradas pelo módulo de hardware. O segundo fluxo representa a solicitação por parte do smartphone do usuário (cliente MQTT) sobre quais equipamentos estão consumindo energia.

Figura 3. Fluxo de identificação dos equipamentos elétricos e consulta pelo cliente utilizando a ferramenta Node-RED.

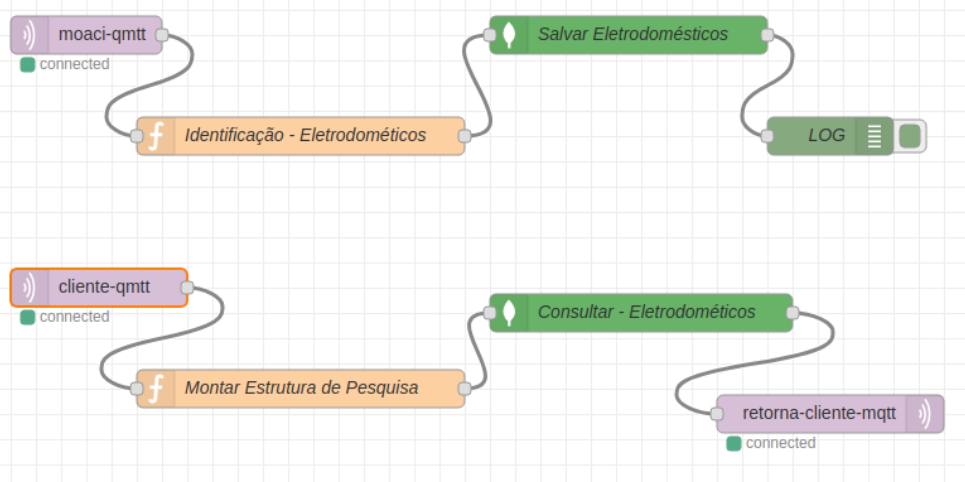

O processo de identificação dos equipamentos elétricos leva em consideração as componentes harmônicas dos sinais de energia capturados: tensão eficaz, corrente eficaz, potência real, potência instantânea, potência reativa e fator de potência. Essas componentes são relacionadas de acordo com as características do equipamento elétrico, verificando se são compatíveis com as componentes medidas obtidas, informando assim se o mesmo está ou não em atividade.

A identificação do tipo de equipamento analisa o comportamento das cargas presentes na rede. As cargas indutivas, geralmente utilizadas em motores e transformadores, criam campos magnéticos pelas bobinas existentes nos equipamentos que estão ligados a ela, produzindo potência reativa com onda de corrente atrasada em relação à tensão. Já as cargas capacitivas, utilizadas em banco de capacitores, lâmpadas fluorescentes e computadores, criam campos elétricos pelos capacitores existentes nestas cargas, provocando um adiantamento da corrente em relação à tensão. Em ambos os casos, o fator de potência é próximo de zero. Por fim, em cargas puramente resistivas, como ferros de passar roupa, chuveiros e lâmpadas incandescentes, é possível observar que a corrente e a 
tensão mudam de polaridade em fase de forma sincronizada, gerando um fator de potência unitário, em que a energia flui em uma mesma direção através do sistema em cada ciclo [Alexander and Sadiku 2013].

\subsection{Aplicativo Android}

No âmbito de permitir o fácil acesso as informações de monitoramento de energia para o consumidor final de forma rápida e intuitiva, foi desenvolvida uma aplicação Android ${ }^{1}$. Na Figura 4 são apresentadas três telas que compõem a aplicação. Na primeira tela o usuário poderá acompanhar, em tempo real, o consumo energético de sua residência e quais equipamentos estão consumindo energia. Já na segunda e terceira tela temos os históricos de consumo energético mensal e diário, respectivamente. Além disso, o usuário pode consultar qual o dia ou mês em que teve mais ou menos consumo de energia a partir de filtros executados nesses históricos.

Figura 4. Telas da aplicação móvel para monitoramento elétrico
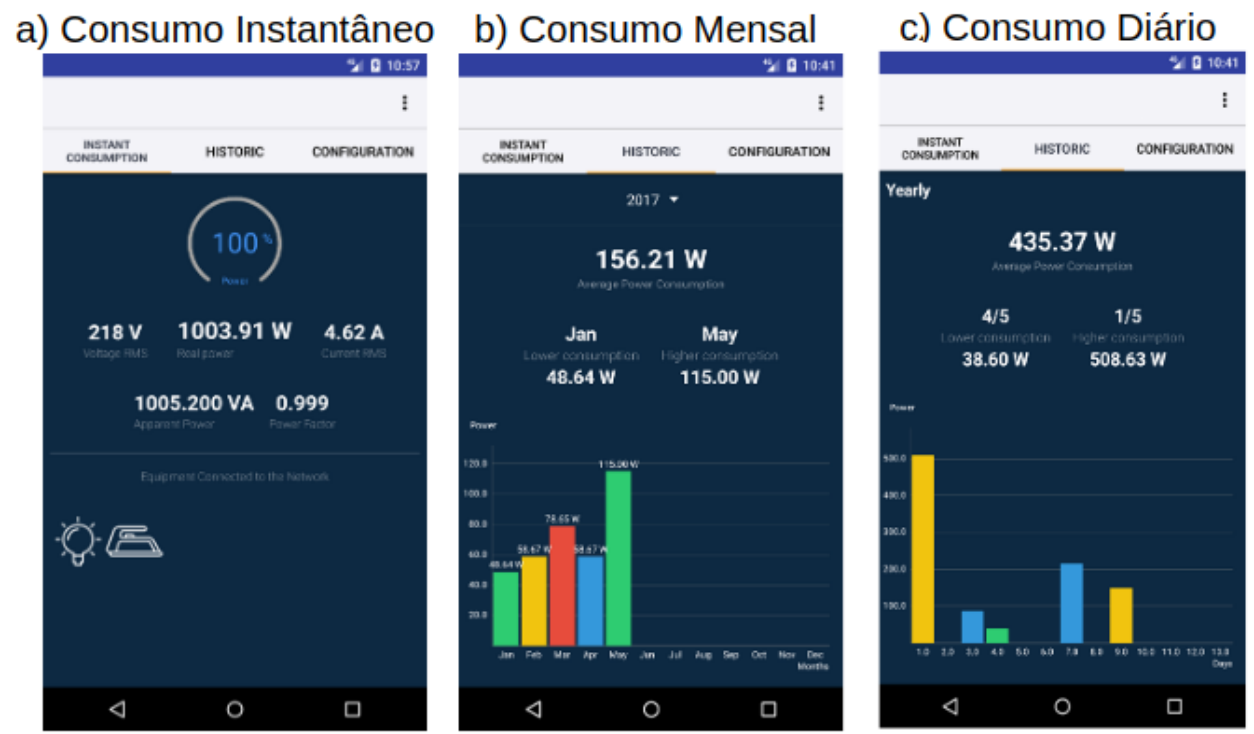

\section{Teste e análise dos resultados}

Para validar o sistema proposto foi desenvolvido um circuito mínimo de teste e diferentes equipamentos elétricos são adicionados ao circuito por etapas. Em cada etapa é realizada a medição das variáveis obtidas, a partir do módulo de hardware descrito previamente. É importante destacar que a alimentação elétrica deste módulo é separada do circuito de teste, com o propósito de evitar possíveis interferências nas medições. Por fim, um amperímetro é adicionado ao circuito como referência para os valores obtidos. Os testes também servem para validar o feedback visual apresentado pelo LED RGB adicionado ao módulo de hardware.

Na Figura 5 é apresentado o primeiro circuito de teste realizado, consistiu da adição de uma lâmpada incandescente ao circuito de teste. É possível evidenciar que o LED RGB apresenta um feedback com a cor amarela simbolizando que existe consumo de energia presente no circuito. Segundo o fabricante da lâmpada, a mesma possui

\footnotetext{
${ }^{1}$ A demonstração da aplicacão móvel está disponivél em https://www.youtube.com/watch?v=bWaXaHJjZSQ
} 
potência de 40 watts, o que a partir da tensão de alimentação de 220 volts, demanda uma corrente de 0.18 amperes. A leitura do amperímetro revelou um valor de 0.21 amperes. Já na aplicação Android evidenciamos que as informações apresentadas possuem uma leve variação em torno de $1 \%$ em torno desse mesmo valor. Além disso, foi observado que o fator de potência apresentado é bem próximo de 1 , confirmando assim a característica de carga da lâmpada, visto que esta é uma carga puramente resistiva.

\section{Figura 5. Circuito de teste com a lâmpada em funcionamento}
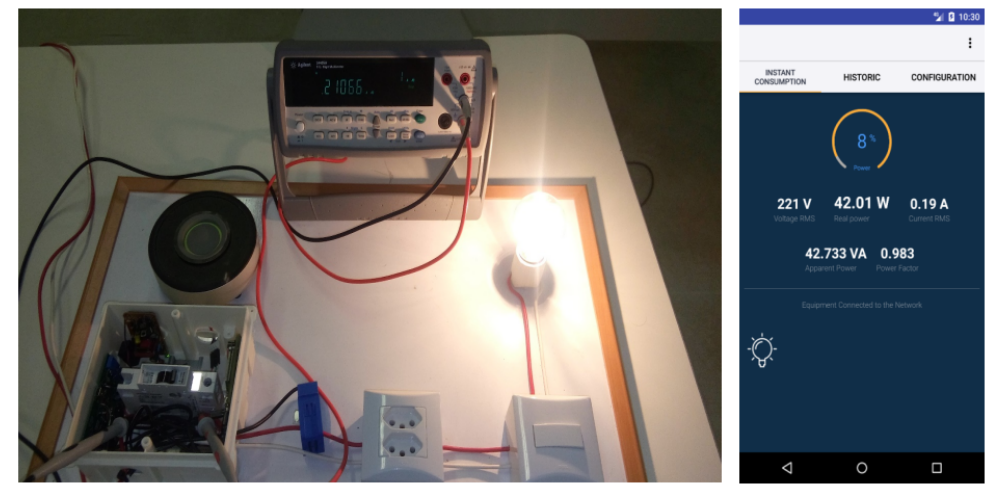

Na Figura 6 é possível visualizar o segundo circuito de teste realizado, consistiu da adição de um ventilador ao circuito de teste. Nesse cenário, o LED RGB apresenta o mesmo feedback visual, ou seja, existe equipamento consumindo energia elétrica na rede. A partir da observação do amperímetro, o ventilador está demandando uma corrente elétrica de 0.34 amperes, valor este bem próximo do que é mostrado no aplicativo Android. $\mathrm{O}$ fator de potência nesse caso se apresenta baixo, confirmando assim a presença do ventilador, dado que é uma carga indutiva.

Figura 6. Circuito de teste com a ventilador em funcionamento
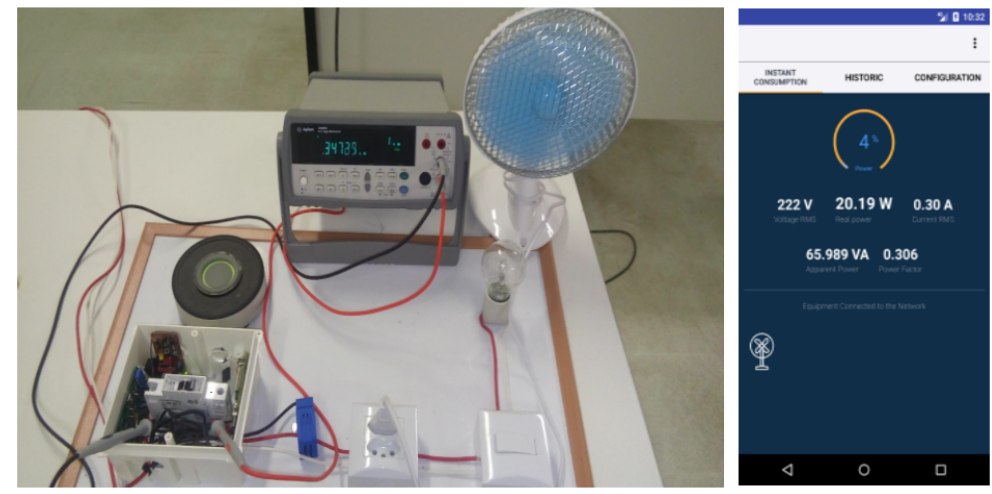

Na Figura 7 é apresentado o último caso de teste, consistindo da adição de um ferro de passar roupa ao circuito de teste. Nesse ambiente, o LED RGB apresenta o feedback visual de cor vermelha, alertando que o consumo está muito alto. Uma vez que o ferro de passar roupa consome cerca de 970 watts e demanda, segundo o amperímetro, uma corrente elétrica de 4.5 amperes. Neste circuito, assim como com o exemplo da lâmpada, temos que ferro de passar roupa também consiste de uma carga resistiva. Essa característica é nítida pelo fato do fator de potência se apresentar quase unitário. 


\section{Figura 7. Circuito de teste com a ferro de passar roupa em funcionamento}
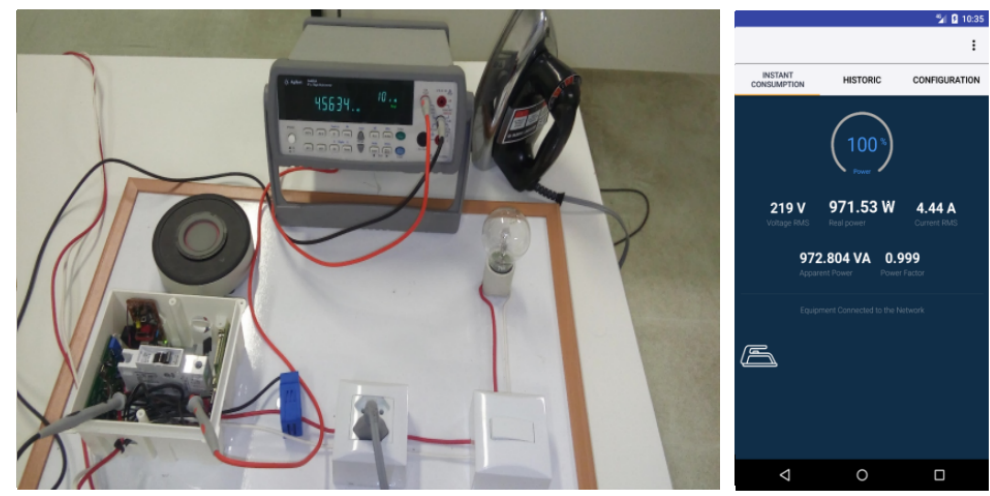

Para permitir uma análise mais fácil dos testes realizadas, a Tabela 1 apresenta os valores de corrente informadas pelo fabricante de cada equipamento adicionado ao circuito de teste, as medidas aferidas pelo amperímetro e as obtidas pelo sistema de monitoramento proposto. Observando os valores de consumo e a identificação dos equipamentos percebe-se a viabilidade da solução como um sistema de monitoramento elétrico residencial.

Tabela 1. Valores de corrente elétrica obtidos a partir da adição dos equipamentos elétricos no circuito de teste

\begin{tabular}{|c|c|c|c|c|c|}
\hline $\begin{array}{c}\text { Equipamento } \\
\text { elétrico }\end{array}$ & $\begin{array}{c}\text { Medida } \\
\text { apresentada } \\
\text { pelo fabricante }\end{array}$ & $\begin{array}{c}\text { Medida aferida } \\
\text { pelo amperimetro }\end{array}$ & $\begin{array}{c}\text { Variaçāo da } \\
\text { leitura do } \\
\text { amperimetro }\end{array}$ & $\begin{array}{c}\text { Medida } \\
\text { apresentada pela } \\
\text { aplicaçāo móvel }\end{array}$ & $\begin{array}{c}\text { Variaçāo da medida } \\
\text { apresentada na } \\
\text { aplicaçāo móvel }\end{array}$ \\
\hline Lâmpada & $0.18 \mathrm{~A}$ & $0.21 \mathrm{~A}$ & $1 \%$ & $0.19 \mathrm{~A}$ & $3 \%$ \\
\hline Ventilador & $0.32 \mathrm{~A}$ & $0.34 \mathrm{~A}$ & $2 \%$ & $0.30 \mathrm{~A}$ & $2 \%$ \\
\hline $\begin{array}{c}\text { Ferro de } \\
\text { passar roupa }\end{array}$ & $4.50 \mathrm{~A}$ & $5.56 \mathrm{~A}$ & $6 \%$ & $4.44 \mathrm{~A}$ & $6 \%$ \\
\hline
\end{tabular}

\section{Conclusão}

O desperdício de energia elétrica, sobretudo nas residências, é um dos grandes problemas enérgicos enfrentados no mundo. O uso de tecnologias do tipo Smart Home Energy Management é de suma importância para minimizar tal problema. No entanto, poucos equipamentos residenciais dispõem de tecnologias que sejam capaz de fornecer informações referentes ao consumo energético. Nesse contexto, este trabalho apresentou um produto mínimo viável de um sistema de gerenciamento energético inteligente, capaz de extrair as informações de consumo dos equipamentos elétricos presentes em uma residência através de um único ponto de coleta.

Os testes realizados demonstram a eficiência e a viabilidade do sistema proposto em monitorar o consumo energético, bem como na identificação dos equipamentos que estão ligados na rede elétrica. Além disso, a possibilidade da visualização dessas informações através da aplicação móvel e pelo LED RGB permitem um melhor gerenciamento de energia por parte do usuário final.

Como trabalhos futuros, nós pretendemos introduzir algoritmos de identificação baseado em técnicas de Machine Learning, permitindo uma maior acurácia na 
identificação e classificação dos equipamentos de acordo com o consumo energético. Também é necessario analisar o fato de se utilizar um ponto centralizado nesta solução, o que pode dificultar a identificação em um cenário com eletrodomésticos iguais na mesma casa (e.g. 2 ventiladores, 2 TVs, etc).

\section{Referências}

ABNT, N. (2004). 5410: Instalações elétricas de baixa tensão. Rio de janeiro.

Alexander, C. K. and Sadiku, M. N. (2013). Fundamentos de circuitos eléctricos (5a. McGraw Hill Mexico.

Andreoli, A. L. (2005). Controlador de demanda e fator de potência de baixo custo para unidades consumidoras de energia elétrica.

Jaffey, T. (2014). Mqtt and coap, iot protocols. Feb-2014.[Online]. Available: http://www. eclipse. org/community/eclipse_newsletter/2014/february/article2. php.[Accessed: 24Mar-2016].

Khan, I., Mahmood, A., Javaid, N., Razzaq, S., Khan, R., and Ilahi, M. (2013). Home energy management systems in future smart grids. J Basic Appl Sci Res, 3.

Lin, C. M. and Chen, M. T. (2017). Design and implementation of a smart home energy saving system with active loading feature identification and power management. In Proc. IEEE 3rd Int. Future Energy Electronics Conf. and ECCE Asia (IFEEC 2017 ECCE Asia), pages 739-742.

Nilsson, A., Wester, M., Lazarevic, D., and Brandt, N. (2018). Smart homes, home energy management systems and real-time feedback: Lessons for influencing household energy consumption from a swedish field study. Energy and Buildings, 179:1525 .

Nyquist, H. (1928). Certain topics in telegraph transmission theory. Transactions of the American Institute of Electrical Engineers, 47(2):617-644.

Pau, G., Collotta, M., Ruano, A., and Qin, J. (2017). Smart home energy management.

Pilloni, V., Floris, A., Meloni, A., and Atzori, L. (2018). Smart home energy management including renewable sources: A qoe-driven approach. IEEE Transactions on Smart Grid, 9(3):2006-2018.

Ramos, M. and Andrade, V. S. (2015). Desenvolvimento, construção e calibração de uma central de monitoramento de consumo de energia elétrica e de água utilizando o microcontrolador arduino. Anais do ENEDS.

Santos, B. P., Silva, L., Celes, C., Borges, J. B., Neto, B. S. P., Vieira, M. A. M., Vieira, L. F. M., Goussevskaia, O. N., and Loureiro, A. (2016). Internet das coisas: da teoria à prática. Minicursos SBRC-Simpósio Brasileiro de Redes de Computadores e Sistemas Distribuidos.

Siqueira, W. V. B. (2014). O microcontrolador arduino como uma central de monitoramento de consumo de energia elétrica. Monografia de conclusão de curso de bacharelado, Instituto Federal de Educação, Ciência e Tecnologia de Minas Gerais, Governador Valadares, MG, Brasil. 